Supporting Information: Details on kinetics measurements; Cartesian coordinates and total energies of optimized structures (minima and TSs on $\mathrm{S}_{0}$ and $\mathrm{T}_{1}$ ) using ONIOM=[UB3LYP/6-311+G(d,p):UB3LYP/631G(d)]; X-ray/ONIOM structures; Tables of X-ray data for trans-1,1'-biindanylidene consisting of crystallographic parameters, positional parameters, bond distances, bond angles, and torsional angles.

\title{
Biindanylidenes: Role of Central Bond Torsion in Nonvertical Triplet Excitation Transfer to the Stilbenes
}

Jack Saltiel, * Jonathan E. Mace, Lucas P. Watkins, David A. Gormin, Ronald J. Clark, and Olga Dmitrenko ${ }^{\dagger}$

Department of Chemistry and Biochemistry, Florida State University, Tallahassee, FL 32306-4390. 
Laser Spectroscopic Measurements. For transient decay measurements, the sidearm cell was placed in a cell holder maintained at the desired $T$ within $\pm 0.1^{\circ} \mathrm{C}$ with use of a Neslab-RTE 4DD constant- $T$ circulation bath. An Omega Engineering model 199 RTD digital thermometer was used to measure solution $T$ in an open cell identically placed in the cell holder. The sample was excited with $378 \mathrm{~nm}$ pulses, and transient absorption was measured perpendicular to the laser pulse using a xenon arc lamp with the monochromator set at $430 \mathrm{~nm}$. Triplet $\mathbf{A}\left({ }^{3} \mathbf{A}^{*}\right)$ decay curves, collected on a LeCroy model 9410 dualchannel digital oscilloscope, were transferred to a PC. Delayed A fluorescence was eliminated from transient absorption signals by subtraction of signals measured without the monitoring light. ${ }^{1,2}$ Data were least squares fitted to mixed first and second order decay ${ }^{1,2}$ on a Dell Precision $5301.4 \mathrm{GHz}$ computer in the MATLAB 6.1 (release 12.1, Optimization Toolbox version 2.1) environment.

\section{References}

(1) Saltiel, J.; Marchand, G. R.; Dabestani, R.; Pecha, J. M. Chem. Phys. Lett. 1983, 100, 219-222.

(2) (2) (a) Saltiel, J.; Marchand, G. R.; Smothers, W. K.; Stout, S. A.; Charlton, J. L. J. Am. Chem. Soc. 1981, 103, 7159-7164. (b) Charlton, J. L.; Dabestani, R.; Saltiel, J. J. Am. Chem. Soc. 1983, $105,3473-3476$. 
c-Bi $\mathrm{S}_{0} \min E=-695.64649$ a.u.

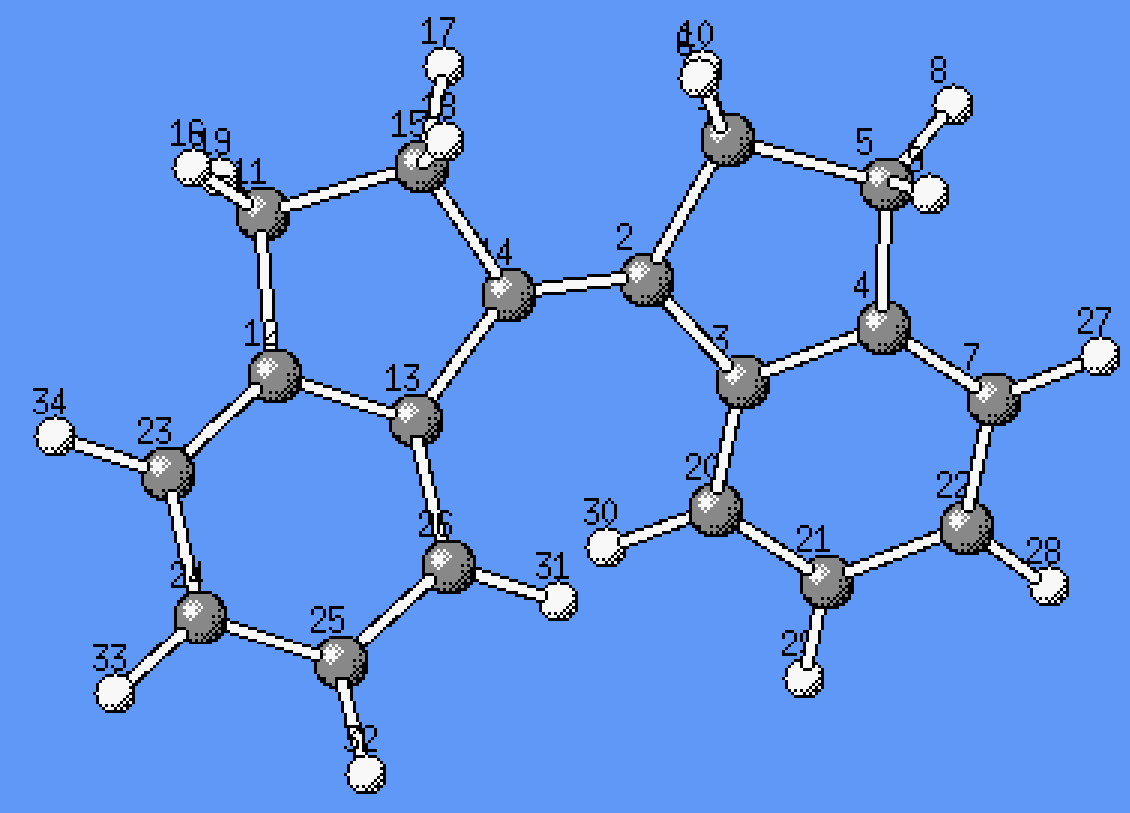

$\begin{array}{llccc}1 & \mathrm{C} & -0.086714 & -1.492422 & 2.284685 \\ 2 & \mathrm{C} & 0.009851 & -0.675892 & 0.9967 \\ 3 & \mathrm{C} & 0.110851 & -1.661922 & -0.096181 \\ 4 & \mathrm{C} & -0.238493 & -2.938519 & 0.382842 \\ 5 & \mathrm{C} & -0.633856 & -2.868824 & 1.838621 \\ 6 & \mathrm{H} & -0.713183 & -1.013409 & 3.039019 \\ 7 & \mathrm{C} & -0.177047 & -4.057741 & -0.442348 \\ 8 & \mathrm{H} & -0.235262 & -3.701085 & 2.429482 \\ 9 & \mathrm{H} & -1.727815 & -2.898717 & 1.945033 \\ 10 & \mathrm{H} & 0.912049 & -1.615252 & 2.72355 \\ 11 & \mathrm{C} & 0.6341 & 2.868688 & 1.838424 \\ 12 & \mathrm{C} & 0.238689 & 2.938454 & 0.382659 \\ 13 & \mathrm{C} & -0.111151 & 1.661951 & -0.096252 \\ 14 & \mathrm{C} & -0.010374 & 0.675958 & 0.996679 \\ 15 & \mathrm{C} & 0.086349 & 1.492561 & 2.284601 \\ 16 & \mathrm{H} & 0.235946 & 3.701176 & 2.42926 \\ 17 & \mathrm{H} & 0.712517 & 1.013387 & 3.03908 \\ 18 & \mathrm{H} & -0.912429 & 1.615875 & 2.723299 \\ 19 & \mathrm{H} & 1.728082 & 2.898046 & 1.944758 \\ 20 & \mathrm{C} & 0.582901 & -1.530803 & -1.411257 \\ 21 & \mathrm{C} & 0.652834 & -2.652778 & -2.231579 \\ 22 & \mathrm{C} & 0.26354 & -3.911416 & -1.760018 \\ 23 & \mathrm{C} & 0.177646 & 4.057626 & -0.442626 \\ 24 & \mathrm{C} & -0.26305 & 3.911352 & -1.760268 \\ 25 & \mathrm{C} & -0.652859 & 2.652826 & -2.231704 \\ 26 & \mathrm{C} & -0.583321 & 1.530893 & -1.411285 \\ 27 & \mathrm{H} & -0.454082 & -5.038274 & -0.061558 \\ 28 & \mathrm{H} & 0.323106 & -4.778186 & -2.412939 \\ 29 & \mathrm{H} & 1.026157 & -2.550482 & -3.247409 \\ 30 & \mathrm{H} & 0.922031 & -0.57205 & -1.779581\end{array}$




$\begin{array}{llrll}31 & \mathrm{H} & -0.922913 & 0.572247 & -1.77947 \\ 32 & \mathrm{H} & -1.026318 & 2.550599 & -3.247491 \\ 33 & \mathrm{H} & -0.322313 & 4.778088 & -2.41326 \\ 34 & \mathrm{H} & 0.455063 & 5.038088 & -0.061933\end{array}$

$\boldsymbol{t}$-Bi $\mathrm{S}_{0} \min E=-695.65137$ a.u.

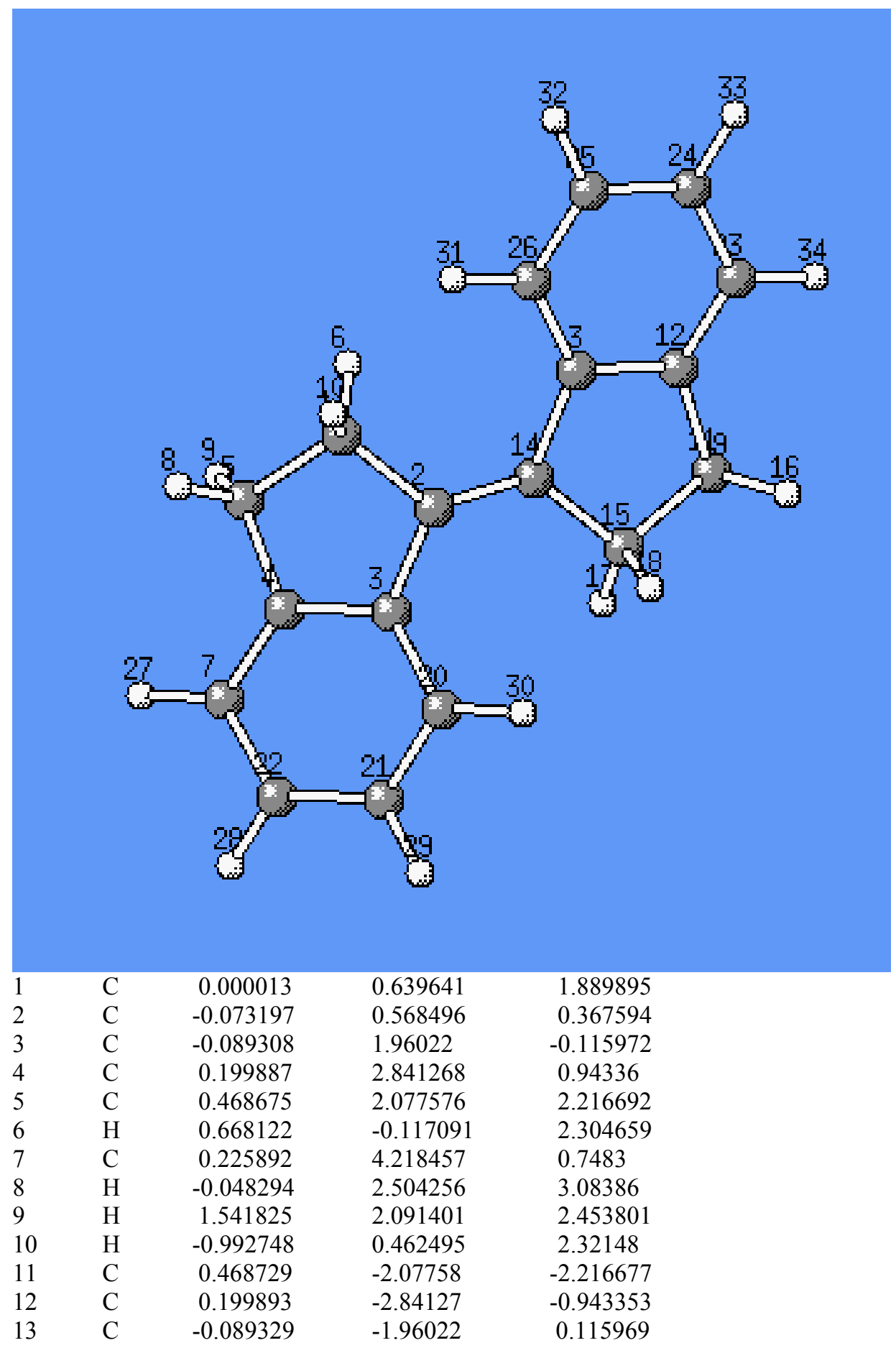




$\begin{array}{llccc}14 & \mathrm{C} & -0.07319 & -0.568496 & -0.367596 \\ 15 & \mathrm{C} & 0.000068 & -0.639642 & -1.889896 \\ 16 & \mathrm{H} & -0.048216 & -2.504256 & -3.083862 \\ 17 & \mathrm{H} & 0.668197 & 0.117085 & -2.304638 \\ 18 & \mathrm{H} & -0.992677 & -0.462487 & -2.321512 \\ 19 & \mathrm{H} & 1.541887 & -2.091414 & -2.453752 \\ 20 & \mathrm{C} & -0.379206 & 2.491621 & -1.384869 \\ 21 & \mathrm{C} & -0.357622 & 3.870339 & -1.57525 \\ 22 & \mathrm{C} & -0.052624 & 4.735503 & -0.519687 \\ 23 & \mathrm{C} & 0.225881 & -4.218459 & -0.748293 \\ 24 & \mathrm{C} & -0.052681 & -4.735502 & 0.519686 \\ 25 & \mathrm{C} & -0.357706 & -3.870335 & 1.575239 \\ 26 & \mathrm{C} & -0.379273 & -2.491617 & 1.384856 \\ 27 & \mathrm{H} & 0.453248 & 4.887064 & 1.575683 \\ 28 & \mathrm{H} & -0.042565 & 5.809853 & -0.683297 \\ 29 & \mathrm{H} & -0.589871 & 4.278378 & -2.555703 \\ 30 & \mathrm{H} & -0.644433 & 1.845261 & -2.209338 \\ 31 & \mathrm{H} & -0.644522 & -1.845255 & 2.209317 \\ 32 & \mathrm{H} & -0.58999 & -4.278372 & 2.555684 \\ 33 & \mathrm{H} & -0.042635 & -5.809852 & 0.683296 \\ 34 & \mathrm{H} & 0.453258 & -4.887067 & -1.575668\end{array}$

Table S3. ${ }^{1}$ p-Bi $\mathrm{S}_{0}$ TS $E=-695.58592$ a.u.

$\begin{array}{lrrrll}0 & 1 & & & & \\ \mathrm{C} & 0 & 1.400796 & 1.124965 & 1.662380 \mathrm{H} & \\ \mathrm{C} & 0 & 0.271097 & 0.676978 & 0.751935 \mathrm{H} & \\ \mathrm{C} & 0 & -0.173437 & 1.776346 & -0.026333 \mathrm{H} & \\ \mathrm{C} & 0 & 0.573183 & 2.945593 & 0.287630 \mathrm{~L} \mathrm{H} & 3 \\ \mathrm{C} & 0 & 1.601731 & 2.635311 & 1.353329 \mathrm{~L} \mathrm{H} & 1 \\ \mathrm{H} & 0 & 2.308845 & 0.537982 & 1.476510 \mathrm{H} & \\ \mathrm{C} & 0 & 0.309810 & 4.143082 & -0.356515 \mathrm{~L} & \\ \mathrm{H} & 0 & 1.453150 & 3.254069 & 2.247460 \mathrm{~L} & \\ \mathrm{H} & 0 & 2.620003 & 2.844355 & 1.001500 \mathrm{~L} & \\ \mathrm{H} & 0 & 1.147487 & 0.949642 & 2.715794 \mathrm{H} & \\ \mathrm{C} & 0 & -1.601738 & -2.635316 & 1.353311 \mathrm{~L} \mathrm{H} & 15 \\ \mathrm{C} & 0 & -0.573184 & -2.945594 & 0.287615 \mathrm{~L} \mathrm{H} & 13 \\ \mathrm{C} & 0 & 0.173437 & -1.776346 & -0.026339 \mathrm{H} & \\ \mathrm{C} & 0 & -0.271100 & -0.676981 & 0.751931 \mathrm{H} & \\ \mathrm{C} & 0 & -1.400801 & -1.124972 & 1.662371 \mathrm{H} & \\ \mathrm{H} & 0 & -1.453165 & -3.254080 & 2.247438 \mathrm{~L} & \\ \mathrm{H} & 0 & -2.308849 & -0.537987 & 1.476504 \mathrm{H} & \\ \mathrm{H} & 0 & -1.147492 & -0.949656 & 2.715787 \mathrm{H} & \\ \mathrm{H} & 0 & -2.620009 & -2.844355 & 1.001474 \mathrm{~L} & \\ \mathrm{C} & 0 & -1.191211 & 1.836862 & -1.007393 \mathrm{H} & \\ \mathrm{C} & 0 & -1.443152 & 3.042959 & -1.643189 \mathrm{~L} \mathrm{H} & 20 \\ \mathrm{C} & 0 & -0.704277 & 4.194712 & -1.327034 \mathrm{~L} & \\ \mathrm{C} & 0 & -0.309809 & -4.143081 & -0.356533 \mathrm{~L} & \\ \mathrm{C} & 0 & 0.704283 & -4.194707 & -1.327047 \mathrm{~L} & \\ \mathrm{C} & 0 & 1.443159 & -3.042953 & -1.643195 \mathrm{LH} & 26 \\ \mathrm{C} & 0 & 1.191215 & -1.836858 & -1.007395 \mathrm{H} & \\ \mathrm{H} & 0 & 0.880104 & 5.038234 & -0.117188 \mathrm{~L} & \\ \mathrm{H} & 0 & -0.917107 & 5.130022 & -1.837248 \mathrm{~L} & \\ \mathrm{H} & 0 & -2.222421 & 3.099933 & -2.399278 \mathrm{~L} & \end{array}$




$$
\begin{aligned}
& \begin{array}{lllll}
\mathrm{H} & 0 & -1.755470 & 0.944787 & -1.256090 \mathrm{H}
\end{array} \\
& \begin{array}{lllll}
\mathrm{H} & 0 & 1.755475 & -0.944783 & -1.256087 \mathrm{H}
\end{array} \\
& \mathrm{H} \quad \begin{array}{lllll}
0 & 2.222431 & -3.099924 & -2.399280 \mathrm{~L}
\end{array} \\
& \mathrm{H} \quad \begin{array}{lllll}
0 & 0.917115 & -5.130015 & -1.837264 \mathrm{~L}
\end{array} \\
& \mathrm{H} \quad \begin{array}{lllll}
0 & -0.880104 & -5.038234 & -0.117212 \mathrm{~L}
\end{array}
\end{aligned}
$$

\begin{tabular}{|c|c|c|c|c|c|c|c|}
\hline $\mathrm{C}$ & 0 & 0.141716 & 1.414513 & 2.201736 & $\mathrm{H}$ & & \\
\hline $\mathrm{C}$ & 0 & 0.016099 & 0.748516 & 0.835986 & $\mathrm{H}$ & & \\
\hline $\mathrm{C}$ & 0 & 0.007923 & 1.762063 & -0.1514 & $\mathrm{H}$ & & \\
\hline $\mathrm{C}$ & 0 & 0.005619 & 3.062487 & 0.472108 & $\mathrm{~L}$ & $\mathrm{H}$ & 3 \\
\hline $\mathrm{C}$ & 0 & -0.044558 & 2.924013 & 1.967606 & $\mathrm{~L}$ & $\mathrm{H}$ & 1 \\
\hline $\mathrm{H}$ & 0 & 1.134836 & 1.202014 & 2.623083 & $\mathrm{H}$ & & \\
\hline $\mathrm{C}$ & 0 & 0.020484 & 4.231597 & -0.260218 & $\mathrm{~L}$ & & \\
\hline $\mathrm{H}$ & 0 & -1.015305 & 3.263102 & 2.355655 & $\mathrm{~L}$ & & \\
\hline $\mathrm{H}$ & 0 & 0.718065 & 3.529117 & 2.471946 & $\mathrm{~L}$ & & \\
\hline $\mathrm{H}$ & 0 & -0.577284 & 1.021279 & 2.924558 & $\mathrm{H}$ & & \\
\hline $\mathrm{C}$ & 0 & 0.044616 & -2.923983 & 1.967704 & $\mathrm{~L}$ & $\mathrm{H}$ & 15 \\
\hline $\mathrm{C}$ & 0 & -0.00552 & -3.062495 & 0.47221 & $\mathrm{~L}$ & $\mathrm{H}$ & 13 \\
\hline $\mathrm{C}$ & 0 & -0.008011 & -1.762093 & -0.151297 & $\mathrm{H}$ & & \\
\hline $\mathrm{C}$ & 0 & -0.016446 & -0.748472 & 0.836091 & $\mathrm{H}$ & & \\
\hline $\mathrm{C}$ & 0 & -0.141836 & -1.414498 & 2.201832 & $\mathrm{H}$ & & \\
\hline $\mathrm{H}$ & 0 & 1.01541 & -3.262927 & 2.35576 & $\mathrm{~L}$ & & \\
\hline $\mathrm{H}$ & 0 & -1.134908 & -1.202069 & 2.623344 & $\mathrm{H}$ & & \\
\hline $\mathrm{H}$ & 0 & 0.577267 & -1.021267 & 2.924533 & $\mathrm{H}$ & & \\
\hline $\mathrm{H}$ & 0 & -0.717916 & -3.529186 & 2.472063 & $\mathrm{~L}$ & & \\
\hline $\mathrm{C}$ & 0 & 0.024077 & 1.744329 & -1.579542 & $\mathrm{H}$ & & \\
\hline $\mathrm{C}$ & 0 & 0.038513 & 2.923552 & $-2.3002 \mathrm{~L}$ & $\mathrm{H}$ & 20 & \\
\hline $\mathrm{C}$ & 0 & 0.036143 & 4.176046 & -1.664476 & $\mathrm{~L}$ & & \\
\hline $\mathrm{C}$ & 0 & -0.020222 & -4.231611 & -0.260115 & $\mathrm{~L}$ & & \\
\hline $\mathrm{C}$ & 0 & -0.035918 & -4.176057 & -1.664371 & $\mathrm{~L}$ & & \\
\hline $\mathrm{C}$ & 0 & -0.038514 & -2.923566 & -2.300097 & $\mathrm{~L}$ & $\mathrm{H}$ & 26 \\
\hline $\mathrm{C}$ & 0 & -0.024249 & -1.744341 & -1.579436 & $\mathrm{H}$ & & \\
\hline $\mathrm{H}$ & 0 & 0.018283 & 5.192876 & 0.249286 & $\mathrm{~L}$ & & \\
\hline $\mathrm{H}$ & 0 & 0.049846 & 5.089668 & -2.251353 & $\mathrm{~L}$ & & \\
\hline $\mathrm{H}$ & 0 & 0.05835 & 2.876258 & -3.386351 & $\mathrm{~L}$ & & \\
\hline $\mathrm{H}$ & 0 & 0.052853 & 0.815753 & -2.119635 & $\mathrm{H}$ & & \\
\hline $\mathrm{H}$ & 0 & -0.053206 & -0.815757 & -2.119507 & $\mathrm{H}$ & & \\
\hline $\mathrm{H}$ & 0 & -0.058404 & -2.876278 & -3.386248 & $\mathrm{~L}$ & & \\
\hline $\mathrm{H}$ & 0 & -0.049494 & -5.089682 & -2.251248 & $\mathrm{~L}$ & & \\
\hline $\mathrm{H}$ & 0 & -0.017876 & -5.192891 & 0.249387 & $\mathrm{~L}$ & & \\
\hline
\end{tabular}

Table S4. $\boldsymbol{c}$-Bi $\mathrm{T}_{1} \mathrm{TS} E=-695.56172$ a.u. 
Table S5. $\boldsymbol{t}$-Bi $\mathrm{T}_{1} \mathrm{TS} E=-695.57218$ a.u.

$\begin{array}{lrrrll}03 & & & & & \\ \mathrm{C} & 0 & -0.674065 & 1.894643 & 0.000542 \mathrm{H} & \\ \mathrm{C} & 0 & -0.635654 & 0.378949 & 0.000063 \mathrm{H} & \\ \mathrm{C} & 0 & -1.953360 & -0.119417 & -0.000105 \mathrm{H} & \\ \mathrm{C} & 0 & -2.898661 & 0.966719 & 0.000030 \mathrm{~L} \mathrm{H} & 3 \\ \mathrm{C} & 0 & -2.170434 & 2.284662 & -0.000060 \mathrm{~L} \mathrm{H} & 1 \\ \mathrm{H} & 0 & -0.153495 & 2.298682 & -0.874759 \mathrm{H} & \\ \mathrm{C} & 0 & -4.260174 & 0.746005 & 0.000090 \mathrm{~L} & \\ \mathrm{H} & 0 & -2.432564 & 2.889951 & 0.877143 \mathrm{~L} & \\ \mathrm{H} & 0 & -2.431902 & 2.889266 & -0.877944 \mathrm{~L} & \\ \mathrm{H} & 0 & -0.154411 & 2.297953 & 0.876743 \mathrm{H} & \\ \mathrm{C} & 0 & 2.170433 & -2.284662 & -0.000068 \mathrm{~L} \mathrm{H} & 15 \\ \mathrm{C} & 0 & 2.898660 & -0.966719 & 0.000028 \mathrm{~L} \mathrm{H} & 13 \\ \mathrm{C} & 0 & 1.953360 & 0.119417 & -0.000107 \mathrm{H} & \\ \mathrm{C} & 0 & 0.635654 & -0.378948 & 0.000064 \mathrm{H} & \\ \mathrm{C} & 0 & 0.674064 & -1.894642 & 0.000553 \mathrm{H} & \\ \mathrm{H} & 0 & 2.432573 & -2.889963 & 0.877123 \mathrm{~L} & \\ \mathrm{H} & 0 & 0.153479 & -2.298691 & -0.874733 \mathrm{H} & \\ \mathrm{H} & 0 & 0.154425 & -2.297942 & 0.876769 \mathrm{H} & \\ \mathrm{H} & 0 & 2.431891 & -2.889254 & -0.877964 \mathrm{~L} & \\ \mathrm{C} & 0 & -2.481919 & -1.451284 & -0.000324 \mathrm{H} & \\ \mathrm{C} & 0 & -3.848526 & -1.650732 & -0.000275 \mathrm{~L} \mathrm{H} & 20 \\ \mathrm{C} & 0 & -4.752505 & -0.572234 & -0.000046 \mathrm{~L} & \\ \mathrm{C} & 0 & 4.260174 & -0.746006 & 0.000091 \mathrm{~L} & \\ \mathrm{C} & 0 & 4.752505 & 0.572233 & -0.000043 \mathrm{~L} & \\ \mathrm{C} & 0 & 3.848527 & 1.650732 & -0.000274 \mathrm{~L} \mathrm{H} & 26 \\ \mathrm{C} & 0 & 2.481920 & 1.451284 & -0.000325 \mathrm{H} & \\ \mathrm{H} & 0 & -4.951486 & 1.586145 & 0.000213 \mathrm{~L} & \\ \mathrm{H} & 0 & -5.822655 & -0.756845 & 0.000002 \mathrm{~L} & \\ \mathrm{H} & 0 & -4.234706 & -2.667301 & -0.000416 \mathrm{~L} & \\ \mathrm{H} & 0 & -1.825252 & -2.307509 & -0.000491 \mathrm{H} & \\ \mathrm{H} & 0 & 1.825253 & 2.307510 & -0.000491 \mathrm{H} & \\ \mathrm{H} & 0 & 4.234707 & 2.667300 & -0.000414 \mathrm{~L} & \\ \mathrm{H} & 0 & 5.822656 & 0.756843 & 0.000007 \mathrm{~L} & \\ \mathrm{H} & 0 & 4.951485 & -1.586146 & 0.000214 \mathrm{~L} & \\ & & & & & \end{array}$


Table S6. ${ }^{3}$ p-Bi $\mathrm{T}_{1} \min E=-695.58528$ a.u.

$\begin{array}{lrrrll}03 & & & & & \\ \mathrm{C} & 0 & -1.332612 & -1.171917 & 1.742110 \mathrm{H} & \\ \mathrm{C} & 0 & -0.255235 & -0.683583 & 0.791093 \mathrm{H} & \\ \mathrm{C} & 0 & 0.179377 & -1.757444 & -0.024758 \mathrm{H} & \\ \mathrm{C} & 0 & -0.531887 & -2.947726 & 0.294838 \mathrm{~L} \mathrm{H} & 3 \\ \mathrm{C} & 0 & -1.525293 & -2.677119 & 1.403790 \mathrm{~L} \mathrm{H} & 1 \\ \mathrm{H} & 0 & -2.257147 & -0.595055 & 1.616137 \mathrm{H} & \\ \mathrm{C} & 0 & -0.269700 & -4.127447 & -0.381286 \mathrm{~L} & \\ \mathrm{H} & 0 & -1.338662 & -3.315656 & 2.276542 \mathrm{~L} & \\ \mathrm{H} & 0 & -2.552707 & -2.890070 & 1.081967 \mathrm{~L} & \\ \mathrm{H} & 0 & -1.030798 & -1.020310 & 2.786582 \mathrm{H} & \\ \mathrm{C} & 0 & 1.525500 & 2.677058 & 1.403562 \mathrm{~L} \mathrm{H} & 15 \\ \mathrm{C} & 0 & 0.531929 & 2.947714 & 0.294771 \mathrm{~L} \mathrm{H} & 13 \\ \mathrm{C} & 0 & -0.179340 & 1.757430 & -0.024812 \mathrm{H} & \\ \mathrm{C} & 0 & 0.255237 & 0.683588 & 0.791085 \mathrm{H} & \\ \mathrm{C} & 0 & 1.332358 & 1.172019 & 1.742341 \mathrm{H} & \\ \mathrm{H} & 0 & 1.339461 & 3.315949 & 2.276174 \mathrm{~L} & \\ \mathrm{H} & 0 & 2.256809 & 0.594891 & 1.617057 \mathrm{H} & \\ \mathrm{H} & 0 & 1.029986 & 1.020911 & 2.786734 \mathrm{H} & \\ \mathrm{H} & 0 & 2.552900 & 2.889453 & 1.081307 \mathrm{~L} & \\ \mathrm{C} & 0 & 1.161553 & -1.779811 & -1.043639 \mathrm{H} & \\ \mathrm{C} & 0 & 1.412783 & -2.968908 & -1.710615 \mathrm{~L} \mathrm{H} & 20 \\ \mathrm{C} & 0 & 0.708452 & -4.140952 & -1.389520 \mathrm{~L} & \\ \mathrm{C} & 0 & 0.269694 & 4.127445 & -0.381315 \mathrm{~L} & \\ \mathrm{C} & 0 & -0.708486 & 4.140953 & -1.389522 \mathrm{~L} & \\ \mathrm{C} & 0 & -1.412782 & 2.968895 & -1.710641 \mathrm{~L} \mathrm{H} & 26 \\ \mathrm{C} & 0 & -1.161515 & 1.779790 & -1.043694 \mathrm{H} & \\ \mathrm{H} & 0 & -0.812766 & -5.038250 & -0.137713 \mathrm{~L} & \\ \mathrm{H} & 0 & 0.920236 & -5.062306 & -1.924922 \mathrm{~L} & \\ \mathrm{H} & 0 & 2.164814 & -2.996772 & -2.495380 \mathrm{~L} & \\ \mathrm{H} & 0 & 1.700005 & -0.872556 & -1.294566 \mathrm{H} & \\ \mathrm{H} & 0 & -1.699956 & 0.872531 & -1.294630 \mathrm{H} & \\ \mathrm{H} & 0 & -2.164826 & 2.996757 & -2.495395 \mathrm{~L} & \\ \mathrm{H} & 0 & -0.920311 & 5.062317 & -1.924890 \mathrm{~L} & \\ \mathrm{H} & 0 & 0.812752 & 5.038253 & -0.137740 \mathrm{~L} & \\ & & & & & \end{array}$




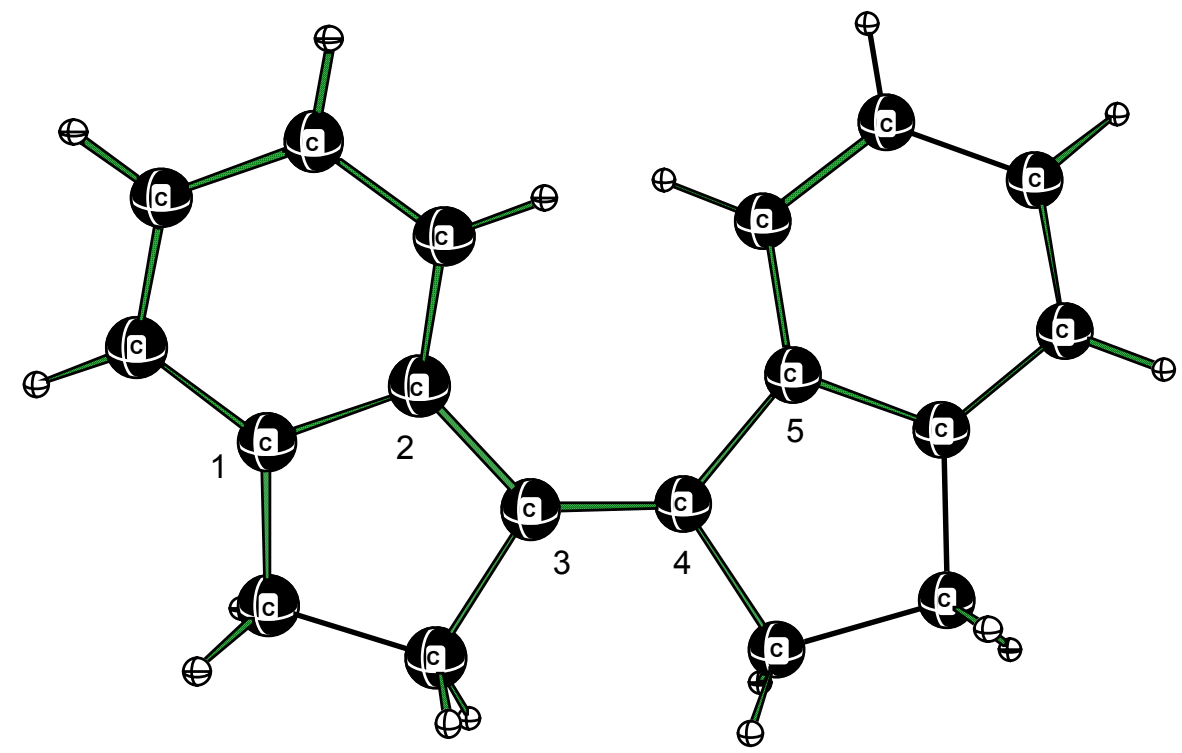

$\mathrm{X}$-ray $\boldsymbol{c}$-Bi (see ref $13 \mathrm{c}$ in manuscript): $<\mathrm{C} 1 \mathrm{C} 2 \mathrm{C} 3 \mathrm{C} 4=163.7^{\circ}$ (one of four angles in two structures in the range of $\left.160.3-167.2^{\circ}\right)<\mathrm{C} 2 \mathrm{C} 3 \mathrm{C} 4 \mathrm{C} 5=-7.9^{\circ}\left(-1^{\circ}\right.$ in the second structure)

In the calculated $\boldsymbol{c}$-Bi $\mathrm{S}_{0} \min :<\mathrm{C} 1 \mathrm{C} 2 \mathrm{C} 3 \mathrm{C} 4=165.6^{\circ}<\mathrm{C} 2 \mathrm{C} 3 \mathrm{C} 4 \mathrm{C} 5=-9.0^{\circ}$

Triplet TS planar $\boldsymbol{c}$-Bi has $<\mathrm{C} 1 \mathrm{C} 2 \mathrm{C} 3 \mathrm{C} 4=177.9^{\circ}<\mathrm{C} 2 \mathrm{C} 3 \mathrm{C} 4 \mathrm{C} 5=3.5^{\circ}$

Phenyl-vinyl angles are fixed to $180^{\circ}$ in $\boldsymbol{c}-\mathrm{Bi}\left(\mathrm{S}_{0}\right): \quad<\mathrm{C} 1 \mathrm{C} 2 \mathrm{C} 3 \mathrm{C} 4=180.0^{\circ}$ fixed $<\mathrm{C} 2 \mathrm{C} 3 \mathrm{C} 4 \mathrm{C} 5=23.8^{\circ}$ ! This structure is $1.3 \mathrm{kcal} / \mathrm{mol}$ higher than the $\mathrm{S}_{0} \boldsymbol{c}$-Bi min

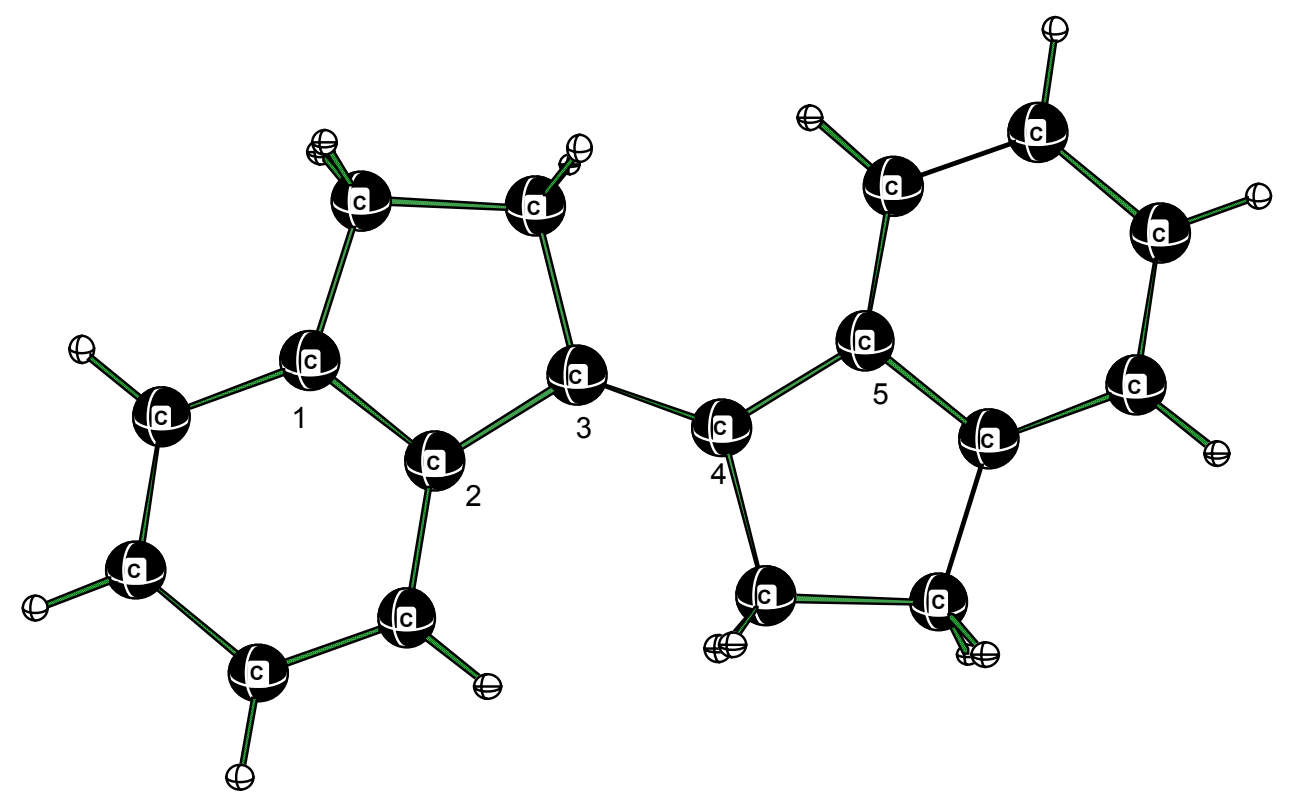

$\mathrm{X}$-ray $\boldsymbol{t}$-Bi (see below and ref $13 \mathrm{~b}$ in manuscript): $<\mathrm{C} 1 \mathrm{C} 2 \mathrm{C} 3 \mathrm{C} 4=179.8^{\circ}<\mathrm{C} 2 \mathrm{C} 3 \mathrm{C} 4 \mathrm{C} 5=180.0^{\circ}$

In the calculated $\boldsymbol{t}$-Bi $\mathrm{S}_{0} \mathrm{~min}:<\mathrm{C} 1 \mathrm{C} 2 \mathrm{C} 3 \mathrm{C} 4=167.6^{\circ}<\mathrm{C} 2 \mathrm{C} 3 \mathrm{C} 4 \mathrm{C} 5=178.4^{\circ}$

Triplet TS planar $\boldsymbol{t}$-Bi has $<\mathrm{C} 1 \mathrm{C} 2 \mathrm{C} 3 \mathrm{C} 4=180.0^{\circ}<\mathrm{C} 2 \mathrm{C} 3 \mathrm{C} 4 \mathrm{C} 5=180.0^{\circ}$

Phenyl-vinyl angles are fixed to $180^{\circ}$ in $\boldsymbol{t}-\mathrm{Bi}\left(\mathrm{S}_{0}\right): \quad<\mathrm{C} 1 \mathrm{C} 2 \mathrm{C} 3 \mathrm{C} 4=180.0^{\circ}$ fixed $<\mathrm{C} 2 \mathrm{C} 3 \mathrm{C} 4 \mathrm{C} 5=180.0^{\circ}$; this structure is $0.5 \mathrm{kcal} / \mathrm{mol}$ higher than $\mathrm{S}_{0} \boldsymbol{t}$ - $\mathbf{B i}$ minimum 
Table S7. Crystal data and structure refinement for $\boldsymbol{t}-\boldsymbol{B} \boldsymbol{B}$.

Identification code

Empirical formula

Formula weight

Temperature

Wavelength

Crystal system

Space group

Unit cell dimensions

Volume

Z

Density (calculated)

Absorption coefficient

$\mathrm{F}(000)$

Crystal size

Theta range for data collection

Index ranges

Reflections collected

Independent reflections

Completeness to theta $=28.23^{\circ}$

Absorption correction

Refinement method

Data / restraints / parameters

Goodness-of-fit on F2

Final R indices [I $>2$ sigma(I)]

$\mathrm{R}$ indices (all data)

Extinction coefficient

Largest diff. peak and hole
$\boldsymbol{t}-\boldsymbol{B i}$

$\mathrm{C}_{18} \mathrm{H}_{16}$

232.31

298(2) K

$0.71073 \AA$

Monoclinic

$\mathrm{P} 2(1) / \mathrm{c}$

$\mathrm{a}=5.3842(16) \AA$

$\alpha=90^{\circ}$.

$\mathrm{b}=5.0639(15) \AA$

$\beta=92.441(6)^{\circ}$.

$\mathrm{c}=22.772(7) \AA$

620.3(3) $\AA^{3}$

2

$1.244 \mathrm{Mg} / \mathrm{m}^{3}$

$0.070 \mathrm{~mm}^{-1}$

248

$0.130 \times 0.290 \times 0.706 \mathrm{~mm}^{3}$

3.58 to $28.23^{\circ}$.

$-7<=\mathrm{h}<=7,-6<=\mathrm{k}<=6,-29<=\mathrm{l}<=30$

8026

$1527[\mathrm{R}(\mathrm{int})=0.0357]$

$99.7 \%$

SADABS

Full-matrix least-squares on $\mathrm{F}^{2}$

1527 / 0 / 115

1.118

$\mathrm{R} 1=0.0568, \mathrm{wR} 2=0.1561$

$\mathrm{R} 1=0.0615, \mathrm{wR} 2=0.1612$

$0.010(11)$

0.303 and -0.267 e. $\AA^{-3}$ 


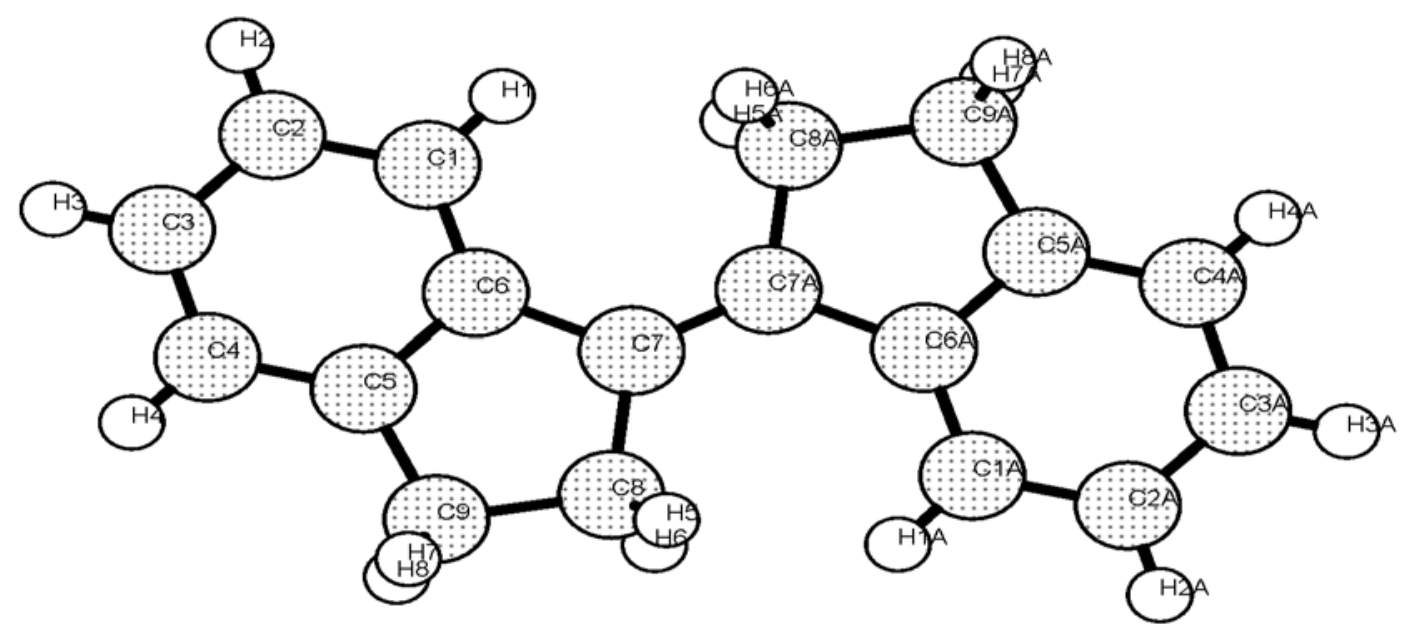

Table S8. Atomic coordinates $\left(\times 10^{4}\right)$ and equivalent isotropic displacement parameters $\left(\AA^{2} \times 10^{3}\right)$ for $\boldsymbol{t}-\boldsymbol{B i}$. $\mathrm{U}(\mathrm{eq})$ is defined as one third of the trace of the orthogonalized Uij tensor.

\begin{tabular}{lrrrr}
\hline & $\mathrm{x}$ & $\mathrm{y}$ & $\mathrm{z}$ & $\mathrm{U}(\mathrm{eq})$ \\
\hline $\mathrm{C}(1)$ & $-864(3)$ & $7106(3)$ & $1047(1)$ & $51(1)$ \\
$\mathrm{C}(2)$ & $-532(3)$ & $6282(4)$ & $1622(1)$ & $60(1)$ \\
$\mathrm{C}(3)$ & $1335(4)$ & $7330(4)$ & $1985(1)$ & $63(1)$ \\
$\mathrm{C}(4)$ & $2883(3)$ & $9268(4)$ & $1775(1)$ & $60(1)$ \\
$\mathrm{C}(5)$ & $2553(3)$ & $10131(3)$ & $1199(1)$ & $46(1)$ \\
$\mathrm{C}(6)$ & $682(2)$ & $9059(2)$ & $826(1)$ & $41(1)$ \\
$\mathrm{C}(7)$ & $734(2)$ & $10284(2)$ & $240(1)$ & $39(1)$ \\
$\mathrm{C}(8)$ & $2787(2)$ & $12369(3)$ & $270(1)$ & $44(1)$ \\
$\mathrm{C}(9)$ & $4003(3)$ & $12209(3)$ & $890(1)$ & $50(1)$ \\
& & & & \\
\hline
\end{tabular}


Table S9. Bond lengths $[\AA]$ and angles $\left[{ }^{\circ}\right]$ for $\boldsymbol{t}$-Bi .

\begin{tabular}{|c|c|}
\hline $\mathrm{C}(1)-\mathrm{C}(2)$ & $1.379(2)$ \\
\hline$C(1)-C(6)$ & $1.400(2)$ \\
\hline $\mathrm{C}(1)-\mathrm{H}(1)$ & $0.97(2)$ \\
\hline$C(2)-C(3)$ & $1.381(3)$ \\
\hline $\mathrm{C}(2)-\mathrm{H}(2)$ & $0.976(19)$ \\
\hline $\mathrm{C}(3)-\mathrm{C}(4)$ & $1.386(3)$ \\
\hline $\mathrm{C}(3)-\mathrm{H}(3)$ & $0.97(2)$ \\
\hline$C(4)-C(5)$ & $1.386(2)$ \\
\hline $\mathrm{C}(4)-\mathrm{H}(4)$ & $0.95(2)$ \\
\hline$C(5)-C(6)$ & $1.3995(19)$ \\
\hline$C(5)-C(9)$ & $1.503(2)$ \\
\hline$C(6)-C(7)$ & $1.4736(19)$ \\
\hline$C(7)-C(7 A)$ & $1.351(2)$ \\
\hline $\mathrm{C}(7)-\mathrm{C}(8)$ & $1.5286(17)$ \\
\hline $\mathrm{C}(8)-\mathrm{C}(9)$ & $1.533(2)$ \\
\hline $\mathrm{C}(8)-\mathrm{H}(5)$ & $1.016(15)$ \\
\hline $\mathrm{C}(8)-\mathrm{H}(6)$ & $0.976(16)$ \\
\hline $\mathrm{C}(9)-\mathrm{H}(7)$ & $0.99(2)$ \\
\hline $\mathrm{C}(9)-\mathrm{H}(8)$ & $1.004(19)$ \\
\hline$C(2)-C(1)-C(6)$ & $119.97(14)$ \\
\hline $\mathrm{C}(2)-\mathrm{C}(1)-\mathrm{H}(1)$ & $119.2(11)$ \\
\hline $\mathrm{C}(6)-\mathrm{C}(1)-\mathrm{H}(1)$ & $120.9(11)$ \\
\hline$C(1)-C(2)-C(3)$ & $120.99(16)$ \\
\hline $\mathrm{C}(1)-\mathrm{C}(2)-\mathrm{H}(2)$ & $118.9(11)$ \\
\hline $\mathrm{C}(3)-\mathrm{C}(2)-\mathrm{H}(2)$ & $120.1(11)$ \\
\hline$C(2)-C(3)-C(4)$ & $119.86(16)$ \\
\hline $\mathrm{C}(2)-\mathrm{C}(3)-\mathrm{H}(3)$ & $120.8(12)$ \\
\hline $\mathrm{C}(4)-\mathrm{C}(3)-\mathrm{H}(3)$ & $119.3(12)$ \\
\hline$C(5)-C(4)-C(3)$ & $119.69(15)$ \\
\hline $\mathrm{C}(5)-\mathrm{C}(4)-\mathrm{H}(4)$ & $120.0(12)$ \\
\hline $\mathrm{C}(3)-\mathrm{C}(4)-\mathrm{H}(4)$ & $120.3(12)$ \\
\hline$C(4)-C(5)-C(6)$ & $120.81(14)$ \\
\hline$C(4)-C(5)-C(9)$ & $127.99(14)$ \\
\hline$C(6)-C(5)-C(9)$ & $111.20(13)$ \\
\hline
\end{tabular}




$\begin{array}{ll}\mathrm{C}(5)-\mathrm{C}(6)-\mathrm{C}(1) & 118.68(13) \\ \mathrm{C}(5)-\mathrm{C}(6)-\mathrm{C}(7) & 110.27(12) \\ \mathrm{C}(1)-\mathrm{C}(6)-\mathrm{C}(7) & 131.06(12) \\ \mathrm{C}(7 \mathrm{~A})-\mathrm{C}(7)-\mathrm{C}(6) & 127.60(14) \\ \mathrm{C}(7 \mathrm{~A})-\mathrm{C}(7)-\mathrm{C}(8) & 125.45(15) \\ \mathrm{C}(6)-\mathrm{C}(7)-\mathrm{C}(8) & 106.95(11) \\ \mathrm{C}(7)-\mathrm{C}(8)-\mathrm{C}(9) & 106.52(11) \\ \mathrm{C}(7)-\mathrm{C}(8)-\mathrm{H}(5) & 110.1(9) \\ \mathrm{C}(9)-\mathrm{C}(8)-\mathrm{H}(5) & 110.9(9) \\ \mathrm{C}(7)-\mathrm{C}(8)-\mathrm{H}(6) & 110.5(10) \\ \mathrm{C}(9)-\mathrm{C}(8)-\mathrm{H}(6) & 109.7(10) \\ \mathrm{H}(5)-\mathrm{C}(8)-\mathrm{H}(6) & 109.2(13) \\ \mathrm{C}(5)-\mathrm{C}(9)-\mathrm{C}(8) & 105.00(11) \\ \mathrm{C}(5)-\mathrm{C}(9)-\mathrm{H}(7) & 111.0(11) \\ \mathrm{C}(8)-\mathrm{C}(9)-\mathrm{H}(7) & 111.2(11) \\ \mathrm{C}(5)-\mathrm{C}(9)-\mathrm{H}(8) & 108.8(11) \\ \mathrm{C}(8)-\mathrm{C}(9)-\mathrm{H}(8) & 112.6(10) \\ \mathrm{H}(7)-\mathrm{C}(9)-\mathrm{H}(8) & 108.2(15)\end{array}$

Symmetry transformations used to generate equivalent atoms:

$\# 1-x,-y+2,-z$

Table S10. Anisotropic displacement parameters $\left(\AA^{2} \times 10^{3}\right)$ for $\boldsymbol{t}$-Bi. The anisotropic displacement factor exponent takes the form: $-2 \pi^{2}\left[h^{2} a^{* 2} U_{11}+\ldots+2 h k a^{*} b^{*} U_{12}\right]$

\begin{tabular}{lcccccc}
\hline & $\mathrm{U}_{11}$ & $\mathrm{U}_{22}$ & $\mathrm{U}_{33}$ & $\mathrm{U}_{23}$ & $\mathrm{U}_{13}$ & $\mathrm{U}_{12}$ \\
\hline $\mathrm{C}(1)$ & $49(1)$ & $51(1)$ & $54(1)$ & $2(1)$ & $2(1)$ & $-8(1)$ \\
$\mathrm{C}(2)$ & $64(1)$ & $61(1)$ & $56(1)$ & $6(1)$ & $8(1)$ & $-8(1)$ \\
$\mathrm{C}(3)$ & $75(1)$ & $69(1)$ & $46(1)$ & $1(1)$ & $2(1)$ & $1(1)$ \\
$\mathrm{C}(4)$ & $63(1)$ & $65(1)$ & $52(1)$ & $-10(1)$ & $-9(1)$ & $-2(1)$ \\
$\mathrm{C}(5)$ & $43(1)$ & $42(1)$ & $52(1)$ & $-10(1)$ & $0(1)$ & $2(1)$ \\
$\mathrm{C}(6)$ & $38(1)$ & $36(1)$ & $48(1)$ & $-5(1)$ & $2(1)$ & $2(1)$ \\
$\mathrm{C}(7)$ & $34(1)$ & $32(1)$ & $50(1)$ & $-3(1)$ & $3(1)$ & $-2(1)$ \\
$\mathrm{C}(8)$ & $37(1)$ & $36(1)$ & $58(1)$ & $-5(1)$ & $1(1)$ & $-5(1)$ \\
$\mathrm{C}(9)$ & $42(1)$ & $45(1)$ & $61(1)$ & $-9(1)$ & $-4(1)$ & $-6(1)$ \\
& & & & & & \\
\hline
\end{tabular}


Table S11. Hydrogen coordinates ( $\left.\mathrm{x} 10^{4}\right)$ and isotropic displacement parameters $\left(\AA^{2} \mathrm{x}\right.$ $\left.10^{3}\right)$ for $\boldsymbol{t}-\boldsymbol{B i}$.

\begin{tabular}{lrrrr}
\hline & $\mathrm{x}$ & $\mathrm{y}$ & $\mathrm{z}$ & $\mathrm{U}(\mathrm{eq})$ \\
\hline $\mathrm{H}(1)$ & & & & \\
$\mathrm{H}(2)$ & $-2180(40)$ & $6330(40)$ & $802(8)$ & $63(5)$ \\
$\mathrm{H}(3)$ & $-1620(30)$ & $4910(40)$ & $1768(8)$ & $65(5)$ \\
$\mathrm{H}(4)$ & $1540(40)$ & $6780(40)$ & $2391(9)$ & $77(6)$ \\
$\mathrm{H}(5)$ & $4140(40)$ & $10030(40)$ & $2025(9)$ & $70(5)$ \\
$\mathrm{H}(6)$ & $2050(30)$ & $14190(30)$ & $192(7)$ & $45(4)$ \\
$\mathrm{H}(7)$ & $4020(30)$ & $12000(30)$ & $-20(7)$ & $49(4)$ \\
$\mathrm{H}(8)$ & $3920(30)$ & $13930(40)$ & $1095(8)$ & $67(5)$ \\
& $5790(40)$ & $11650(40)$ & $889(8)$ & $61(5)$ \\
\hline
\end{tabular}

Table S12. Torsion angles $\left[^{\circ}\right]$ for $\boldsymbol{t}$-Bi.

\begin{tabular}{llllc}
\hline $\mathrm{C} 6$ & $-\mathrm{C} 1$ & $-\mathrm{C} 2$ & $-\mathrm{C} 3$ & $-0.9(3)$ \\
$\mathrm{C} 2$ & $-\mathrm{C} 1$ & $-\mathrm{C} 6$ & $-\mathrm{C} 5$ & $0.2(2)$ \\
$\mathrm{C} 2$ & $-\mathrm{C} 1$ & $-\mathrm{C} 6$ & $-\mathrm{C} 7$ & $-179.96(14)$ \\
$\mathrm{C} 1$ & $-\mathrm{C} 2$ & $-\mathrm{C} 3$ & $-\mathrm{C} 4$ & $1.0(3)$ \\
$\mathrm{C} 2$ & $-\mathrm{C} 3$ & $-\mathrm{C} 4$ & $-\mathrm{C} 5$ & $-0.3(3)$ \\
$\mathrm{C} 3$ & $-\mathrm{C} 4$ & $-\mathrm{C} 5$ & $-\mathrm{C} 6$ & $-0.3(3)$ \\
$\mathrm{C} 3$ & $-\mathrm{C} 4$ & $-\mathrm{C} 5$ & $-\mathrm{C} 9$ & $179.45(17)$ \\
$\mathrm{C} 4$ & $-\mathrm{C} 5$ & $-\mathrm{C} 6$ & $-\mathrm{C} 1$ & $0.4(2)$ \\
$\mathrm{C} 4$ & $-\mathrm{C} 5$ & $-\mathrm{C} 6$ & $-\mathrm{C} 7$ & $-179.46(14)$ \\
$\mathrm{C} 9$ & $-\mathrm{C} 5$ & $-\mathrm{C} 6$ & $-\mathrm{C} 1$ & $-179.42(12)$ \\
$\mathrm{C} 9$ & $-\mathrm{C} 5$ & $-\mathrm{C} 6$ & $-\mathrm{C} 7$ & $0.71(16)$ \\
$\mathrm{C} 4$ & $-\mathrm{C} 5$ & $-\mathrm{C} 9$ & $-\mathrm{C} 8$ & $-178.85(16)$ \\
$\mathrm{C} 6$ & $-\mathrm{C} 5$ & $-\mathrm{C} 9$ & $-\mathrm{C} 8$ & $0.96(16)$ \\
$\mathrm{C} 1$ & $-\mathrm{C} 6$ & $-\mathrm{C} 7$ & $-\mathrm{C} 8$ & $178.06(13)$ \\
$\mathrm{C} 1$ & $-\mathrm{C} 6$ & $-\mathrm{C} 7$ & $-\mathrm{C} 7 \mathrm{~A}$ & $-1.8(2)$ \\
$\mathrm{C} 5$ & $-\mathrm{C} 6$ & $-\mathrm{C} 7$ & $-\mathrm{C} 8$ & $-2.09(13)$ \\
$\mathrm{C} 5$ & $-\mathrm{C} 6$ & $-\mathrm{C} 7$ & $-\mathrm{C} 7 \mathrm{~A}$ & $178.07(12)$ \\
$\mathrm{C} 6$ & $-\mathrm{C} 7$ & $-\mathrm{C} 8$ & $-\mathrm{C} 9$ & $2.61(13)$ \\
$\mathrm{C} 7 \mathrm{~A}$ & $-\mathrm{C} 7$ & $-\mathrm{C} 8$ & $-\mathrm{C} 9$ & $-177.55(11)$ \\
$\mathrm{C} 6$ & $-\mathrm{C} 7$ & $-\mathrm{C} 7 \mathrm{~A}$ & $-\mathrm{C} 6 \mathrm{~A}$ & $180.00(11)$ \\
$\mathrm{C} 6$ & $-\mathrm{C} 7$ & $-\mathrm{C} 7 \mathrm{~A}$ & $-\mathrm{C} 8 \mathrm{~A}$ & $-0.19(19)$ \\
$\mathrm{C} 8$ & $-\mathrm{C} 7$ & $-\mathrm{C} 7 \mathrm{~A}$ & $-\mathrm{C} 6 \mathrm{~A}$ & $0.19(19)$ \\
$\mathrm{C} 8$ & $-\mathrm{C} 7$ & $-\mathrm{C} 7 \mathrm{~A}$ & $-\mathrm{C} 8 \mathrm{~A}$ & $-180.00(12)$ \\
$\mathrm{C} 7$ & $-\mathrm{C} 8$ & $-\mathrm{C} 9$ & $-\mathrm{C} 5$ & $-2.16(14)$
\end{tabular}

